\title{
A moving boundary solution for solidification of lava lake and magma intrusion in the presence of time-varying contact temperature
}

\author{
AJAY MANGLIK \\ National Geophysical Research Institute, Hyderabad 500 007, India. \\ e-mail: ajay@ngri.res.in_ajay_ngri@yahoo.co.in
}

During the solidification of a lava lake heat is released convectively from the top surface as well as conductively into the country rock from the base, leading to non-uniform solidification. The upper solidified layer grows at a faster rate than the lower solidified layer. Similarly, solidification of magma intrusion within the crust is also non-uniform due to the presence of thermal gradient in the crust. Available analytical solution for solidification of a melt layer assumes only symmetric cooling about the centre of the layer. In the present work a moving boundary solution for thermal evolution and non-uniform solidification of a melt layer incorporating time-varying contact temperature conditions at both of its boundaries is developed. The solution is obtained by using the Fourier spectral approach in the space domain and a modified finite difference scheme in the time domain, and is validated with available analytical solutions for simple cases and a semi-analytical solution for the case involving temperature gradient in the country rock. This solution can be used to analyse solidification of lava lakes and magma intrusions experiencing time-dependent temperature variation at their contacts with the country rock.

\section{Introduction}

Partial melting of upwelling mantle material at plumes generates large volumes of melt (White and McKenzie 1989; Watson and McKenzie 1991). This melt ascends through the lithosphere and, in the case of continental lithosphere, gets trapped mainly at the base of the continental crust forming an underplated layer (Cox 1993). About one fourth of the volume of the fractionated melt erupts on the surface of the Earth in the form of volcanism (White 1993). These underplated and volcanic melts can flow for large lateral distances depending on their viscosity, solidification rate, melt supply rate from the source, and the strength of the medium through which the underplated melt propagates (White 1993). These surface and subsurface flows modify the heat and mass budget of the crust not only at the source region but also at large lateral distances. Solidification of lava lakes of basaltic flows releases heat to the air/water from the top surface and to the underlying basement at the bottom. The underplated melt liberates heat to the crust and mantle during the solidification process. The heat thus liberated affects many geotectonic processes (Gettings 1988; Karner et al 1992). It is, therefore, important to understand the mechanism of solidification of these bodies.

The process of solidification of magma intrusions has been studied by adopting the fluid dynamical approach (Huppert 1990; Lister and Kerr 1991; Huppert and Worster 1992; Worster et al 1993; Lister 1995; Jaupart and Tait 1995) and through transient heat conduction models without (Carslaw and Jaeger 1959; Jaeger 1968) and with the Stefan condition (Lightfoot 1929; Carslaw and Jaeger 1959; Turcotte and Schubert 1982; Delany 1987, 1988;

Keywords. Non-uniform solidification; moving boundary problem; continental crust; time-varying contact temperature. 
Manglik and Singh 1995). Fluid dynamical models have been used to analyse the lateral flow of solidifying lava (Griffiths and Fink 1992; Lister 1995) and to understand the compositional stratification and formation of mush zones within magma chambers (Huppert 1990; Huppert and Worster 1992; Worster et al 1993). This approach has been extended to understand the effect of kinetic undercooling in driving convection in the interior of lava lakes and in the formation of crystals in the regions of the lake remote from the cooled upper boundary layer (Worster et al 1993). In heat conduction models, Jaeger (1968) adjusted the excess heat released during the solidification of intrusion by increasing the apparent specific heat and the apparent temperature of the intrusion. A solution of the Stefan problem was discussed by Carslaw and Jaeger (1959) and Turcotte and Schubert (1982) for a simple case of cooling of a dyke in contact with a constant temperature host. Manglik and Singh (1995) presented a semi-analytical solution of the Stefan problem for a more complicated case where the intrusion conducts heat to the continental crust, having initial geothermal gradient and radiogenic sources, at a non-uniform rate from both the contact surfaces.

The studies carried out using the above approaches highlighted the importance of the nonuniform cooling of an intrusion/lava lake. Worster et al (1993) suggested a reduction in the solidification time of lava lake by a factor of two in the presence of cooling by heat conduction from the base of the lake into the country rock. Manglik and Singh (1995) obtained different contact temperatures at both the surfaces of the intrusion as a result of non-uniform cooling. This effect is pronounced for the lava lakes because of fast convective removal of heat from the upper surface of the lake to the air/water in comparison to slow conductive heat removal from the base to the country rock.

In the present work, a semi-analytical solution for the solidification of a lava lake is developed using the Fourier series method (Gliko and Rovensky 1985; Manglik et al 1992; Manglik and Singh 1995) and by treating the system as consisting of two moving boundaries. These moving boundaries separate the central melt zone from the upper and lower solidified zones of the intrusion. The solution obtained here includes timevarying temperature boundary conditions at both the surfaces of the intrusion, facilitating the use of any type of time variation at the surfaces of the intrusion. This time variation can be constructed depending on the prevailing temperature conditions in the surrounding host rock or can be implicitly obtained by coupling these surfaces with the temperature solutions for the host rock.
The developed semi-analytical solution is validated with the results of cooling half-space and cooling dyke models (Carslaw and Jaeger 1959), and with the underplating model (Manglik and Singh 1995).

\section{Mathematical formulation}

During the solidification of a lava lake convective heat removal maintains the temperature of the upper surface at some fixed value but at the lower surface, which is in contact with the underlying country rock, the flow of heat into the country rock varies with time. We analyse this scenario through a one-dimensional model of transient heat conduction by assuming a melt body of thickness $2 a$ having an initial temperature of $T_{p}$ (figure 1 ). With the onset of the solidification the upper and the lower boundaries of the body are prescribed time-varying temperatures. As the solidification proceeds the body is subdivided into three domains; the upper and lower solidified domains, and the middle melt domain. These domains are separated by moving phase boundaries. We assume that the material properties of the three domains are same, and that the lava lake contains no radiogenic heat sources. Under these assumptions the one-dimensional heat conduction equation in non-dimensional form can be expressed as:

$$
\frac{\partial u_{i}}{\partial t}=\frac{\partial^{2} u_{i}}{\partial z^{2}}
$$

where $i$ is the index number of the domain. $i=1$ and 3 represent the upper and the lower solidified domains, respectively, and $i=2$ represents the melt domain. $u_{i}$ is temperature distribution in the $i$ th domain normalized with respect to the solidus temperature $T_{m}$. The variables $z$ and $t$ are normalized with respect to half thickness of the body $a$

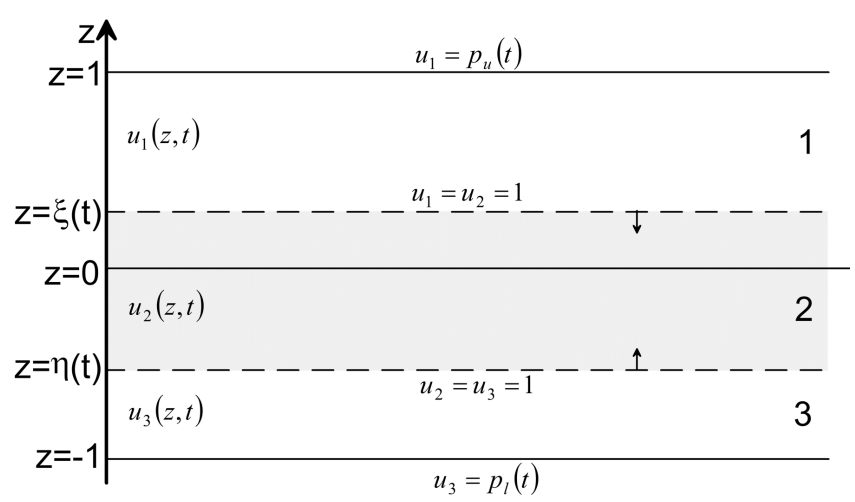

Figure 1. A schematic diagram of one-dimensional model with two moving boundaries and prescribed time-dependent contact temperatures. 
and characteristic time $\tau=a^{2} / \kappa ; \kappa$ is the thermal diffusivity), respectively.

The system of equations (1) is solved with the non-dimensional boundary conditions

$$
\begin{aligned}
u_{1}(1, t) & =p_{u}(t), \\
u_{1}(\xi(t), t) & =u_{2}(\xi(t), t)=1, \\
u_{3}(\eta(t), t) & =u_{2}(\eta(t), t)=1, \\
u_{3}(-1, t) & =p_{l}(t),
\end{aligned}
$$

where $p_{u}(t)$ and $p_{l}(t)$ are normalized upper $(z=1)$ and lower $(z=-1)$ time-varying contact surface temperatures, respectively. $\xi(t)$ and $\eta(t)$ are the positions of the upper and lower moving boundaries, respectively.

Besides these boundary conditions, the Stefan conditions (Carslaw and Jaeger 1959) have been incorporated to adjust for the heat exchange due to melting and solidification. These are

$$
\begin{array}{rr}
-\frac{\partial u_{1}}{\partial z}+\frac{\partial u_{2}}{\partial z}=-\alpha \frac{d \xi}{d t} \quad(z=\xi(t)), \\
\frac{\partial u_{3}}{\partial z}-\frac{\partial u_{2}}{\partial z}=\alpha \frac{d \eta}{d t} \quad(z=\eta(t)),
\end{array}
$$

where $\alpha\left(=L / c T_{m}\right)$ is the normalized Stefan parameter $L$ and $c$ are the latent heat and specific heat, respectively.

\section{Fourier series solution}

The system of equations (1-3) is solved by using the Fourier series method in space domain. In this method, the temperatures in the three domains are first expanded in an appropriate Fourier sine series form such that respective boundary conditions are satisfied and then these expansions are substituted into the heat conduction equation (1). The transformed heat conduction equation for each domain is then reduced to a set of infinite first-order ordinary differential equations (ODEs) in time by applying the property of orthogonality. Since the details of the method are given in Gliko and Rovensky (1985) and Manglik and Singh (1995), intermediate steps of the solution are skipped and only the final solution for all the domains is presented in this section.
In the first domain the temperature

$$
\begin{aligned}
u_{1}(z, t)= & \sum_{k=1}^{\infty} A_{k}(t) \sin \left(k \pi \frac{z-1}{\xi-1}\right)+\left(\frac{z-1}{\xi-1}\right) \\
& -\left[\left(\frac{z-1}{\xi-1}\right)-1\right] p_{u}(t),
\end{aligned}
$$

is used such that the boundary conditions for this domain are satisfied. The heat conduction equation for this domain reduces to a system of ODEs of the form

$$
\begin{aligned}
\frac{d A_{n}}{d t}= & -\left(\frac{n \pi}{\xi-1}\right)^{2} A_{n}(t)-\frac{2}{n \pi} \frac{d p_{u}(t)}{d t}-\frac{2}{\xi-1} \\
& \times \frac{d \xi}{d t}\left[\sum_{k=1}^{\infty} A_{k}(t) P_{n k}+\frac{(-1)^{n}}{n \pi}\left\{1-p_{u}(t)\right\}\right],
\end{aligned}
$$

where

$$
P_{n k}= \begin{cases}n k(-1)^{n+k} /\left(n^{2}-k^{2}\right) & (n \neq k), \\ 1 / 4 & (n=k)\end{cases}
$$

and $A_{n}(t)$ are the coefficients of the Fourier series for the first domain. The initial values of these coefficients are obtained from the initial temperature condition, which gives

$$
A_{n}(0)=\frac{2}{n \pi}\left[1-p_{u}(0)\right]
$$

Similarly the temperature in the lower solidified domain is expanded as

$$
\begin{aligned}
u_{3}(z, t)= & \sum_{k=1}^{\infty} B_{k}(t) \sin \left(k \pi \frac{z+1}{\eta+1}\right)+\left(\frac{z+1}{\eta+1}\right) \\
& -\left[\left(\frac{z+1}{\eta+1}\right)-1\right] p_{l}(t) .
\end{aligned}
$$

In this case the heat conduction equation reduces to the ODEs 


$$
\begin{aligned}
\frac{d B_{n}}{d t}= & -\left(\frac{n \pi}{\eta+1}\right)^{2} B_{n}(t) \\
& -\frac{2}{n \pi} \frac{d p_{l}(t)}{d t}-\frac{2}{\eta+1} \frac{d \eta}{d t} \\
& \times\left[\sum_{k=1}^{\infty} B_{k}(t) P_{n k}+\frac{(-1)^{n}}{n \pi}\left\{1-p_{l}(t)\right\}\right],
\end{aligned}
$$

and the initial values of the Fourier coefficients are obtained as

$$
B_{n}(0)=\frac{2}{n \pi}\left[1-p_{l}(0)\right] .
$$

In the melt domain the transformation

$$
u_{2}(z, t)=1+\sum_{k=1}^{\infty} C_{k}(t) \sin \left(k \pi \frac{z-\eta}{\xi-\eta}\right)
$$

reduces the heat conduction equation to

$$
\begin{aligned}
\frac{d C_{n}}{d t} & =-\left(\frac{n \pi}{\xi-\eta}\right)^{2} C_{n}(t)-\frac{2}{\xi-\eta} \\
& \times\left[\frac{d \xi}{d t} \sum_{k=1}^{\infty} C_{k}(t) P_{n k}-\frac{d \eta}{d t} \sum_{k=1}^{\infty} C_{k}(t) Q_{n k}\right],
\end{aligned}
$$

where

$$
Q_{n k}=\left\{\begin{array}{ll}
k \pi /\left(n^{2}-k^{2}\right) & (n \neq k) \\
1 / 4 & (n=k)
\end{array},\right.
$$

and the initial values of the Fourier coefficients are given by

$$
C_{n}(0)=2\left(u_{p}-1\right)\left[\frac{1-(-1)^{n}}{n \pi}\right]
$$

where $u_{p}$ is normalized initial temperature of the melt zone.

The Stefan conditions at the two moving interfaces also reduce to ODEs form when expressions for $u_{1}, u_{2}$, and $u_{3}$ are substituted into equation (3). These conditions in the Fourier series form are expressed as

$$
\begin{aligned}
\alpha \frac{d \xi}{d t}= & \left(\frac{\pi}{\xi-1}\right) \sum_{k=1}^{\infty}(-1)^{k} k A_{k}(t) \\
& -\left(\frac{\pi}{\xi-\eta}\right) \sum_{k=1}^{\infty}(-1)^{k} k C_{k}(t) \\
& -\left(\frac{1}{\xi-1}\right)\left[p_{u}(t)-1\right],
\end{aligned}
$$

and

$$
\alpha \frac{d \eta}{d t}=\left(\frac{\pi}{\eta+1}\right) \sum_{k=1}^{\infty}(-1)^{k} k B_{k}(t)-\left(\frac{\pi}{\xi-\eta}\right)
$$

$$
\times \sum_{k=1}^{\infty} k C_{k}(t)-\left(\frac{1}{\eta+1}\right)\left[p_{l}(t)-1\right] .
$$

Equations (5, 8, 11, 13, and 14) form a coupled system of first-order ODEs that can be solved iteratively to obtain the positions of solidification fronts and the Fourier coefficients. These values when substituted into equations $(4,7$, and 10$)$ give the evolution of the thermal structure within the lava lake.

The Stefan conditions (equations 13 and 14) are singular at the starting time where $\xi=1$ and $\eta=-1$. Therefore, these equations cannot be directly used to solve the system near the starting time. Under the assumption that there is no superheat in the system, equations (13) and (14) can be rearranged to calculate the movement of solidification fronts at very small time $t \leq \varepsilon$ in the following form

$$
\xi(t)=1-\sqrt{\frac{2}{\alpha}\left[1-\int_{0}^{t} p_{u}(t) d t\right]},
$$

and

$$
\eta(t)=-1+\sqrt{\frac{2}{\alpha}}\left[1-\int_{0}^{t} p_{l}(t) d t\right] .
$$

For time $t>\varepsilon$ the Stefan conditions (equations 13 and 14) were used in subsequent computations. In fact, a single time step was sufficient to overcome the problem of singularity.

\subsection{Thermal evolution after solidification}

As the solidification of the body proceeds the two moving fronts get closer and at time $t=t^{*}$ 
the body becomes completely solidified with the merger of the two phase boundaries. After this time further cooling of the body takes place through pure conduction. Under the assumption that the melt and solid zones have the same material properties, the distinction among the three domains vanishes. Now, a single heat conduction equation

$$
\frac{\partial u}{\partial t}=\frac{\partial^{2} u}{\partial z^{2}},
$$

can be solved with the time-varying boundary conditions

$$
\begin{array}{r}
u(-1, t)=p_{l}(t), \\
u(1, t)=p_{u}(t) .
\end{array}
$$

The initial temperature condition for this case was constructed from the temperature distribution in the two solidified domains at the end of solidification $\left(t=t^{*}\right)$. This is given as

$$
u\left(z, t^{*}\right)=\left\{\begin{array}{ll}
u_{1}\left(z, t^{*}\right) & \xi^{*} \leq z \leq 1 \\
u_{2}\left(z, t^{*}\right) & -1 \leq z \leq \xi^{*}
\end{array},\right.
$$

where $\xi^{*}=\xi\left(t^{*}\right)=\eta\left(t^{*}\right)$.

Following the Fourier series approach equation (17) reduces to the first-order ODEs as

$$
\begin{aligned}
\frac{d C_{n}}{d t}= & -\left(\frac{n \pi}{2}\right)^{2} C_{n}(t)+\frac{2(-1)^{n}}{n \pi} \frac{d p_{u}(t)}{d t} \\
& -\frac{2}{n \pi} \frac{d p_{l}(t)}{d t},
\end{aligned}
$$

with the initial coefficients

$$
\begin{aligned}
C_{n}\left(t^{*}\right)= & \sum_{k=1}^{\infty} B_{k}\left(t^{*}\right) P_{a b}+\sum_{k=1}^{\infty} A_{k}\left(t^{*}\right) Q_{b c} \\
& +\left(\frac{2}{n \pi}\right)^{2}\left[\frac{1-p_{l}}{1+\xi}+\frac{1-p_{u}}{1-\xi}\right] \\
& \times \sin \left(\frac{n \pi}{2}(1+\xi)\right),
\end{aligned}
$$

where

$$
P_{a b}=\left\{\begin{array}{cc}
\frac{1}{2}\left[\frac{\sin \{(a-b)(1+\xi)\}}{a-b}\right. & \\
\left.-\frac{\sin \{(a+b)(1+\xi)\}}{(a+b)}\right] & (a \neq b), \\
\frac{1}{2}\left[(1+\xi)-\frac{\sin \{n \pi(1+\xi)\}}{n \pi}\right] & (a=b)
\end{array}\right.
$$

and

$$
Q_{b c}=\left\{\begin{array}{l}
\frac{1}{2}\left[\left(\frac{1}{c+b}\right) \sin \{(c-b)-(c+b) \xi\}\right. \\
-\left(\frac{1}{c-b}\right) \sin \{(c+b) \\
-(c-b) \xi\}] \quad(b \neq c) \\
-\frac{(-1)^{n}}{2}\left[(1-\xi)+\frac{1}{n \pi} \sin \{n \pi(1+\xi)\}\right] \\
\quad(b=c) .
\end{array}\right.
$$

In these expressions $a=k \pi /(1+\xi), b=n \pi / 2$, $c=k \pi /(1-\xi)$. We solved the above system of nonlinear ODEs in the Fourier coefficients by using a finite difference scheme in time based on the modified Euler's method (Melamed 1958). The infinite summation series of the Fourier coefficients were truncated after the first 50 terms. This number was chosen by performing the relative error analysis with respect to 100 Fourier coefficients.

\section{Results}

In this section we present two examples; one each for solidification of lava lake and magma intrusion.

\subsection{Solidification of lava lake}

We computed the positions of moving fronts and the thermal evolution within the solidifying lava lake at different times by using the present method and compared the results with the analytical solutions of cooling half-space model (CHSM) and finite thickness dyke model (FTDM) (Carslaw and Jaeger 1959). Although the above solution is obtained in non-dimensional form, computational results are presented in dimensional form relevant to geological problems. In the first example, we 
considered a 2-km thick layer which gives the solidification time of the order of m.y. These results, however, can be scaled down to lava lakes of few meters thickness in which case the solidification time would be of the order of days or months. Latent heat $(L)$, specific heat $(c)$, and thermal diffusivity $(\kappa)$ were assigned the values of $330 \mathrm{~kJ} / \mathrm{kg}$, $1.0 \mathrm{~kJ} / \mathrm{kg} \cdot \mathrm{K}$, and $10^{-6} \mathrm{~m}^{2} / \mathrm{s}$, respectively. The body was kept at an initial temperature $T_{m}$ of $1100^{\circ} \mathrm{C}$ and no superheat was considered.

We assigned the upper boundary temperature as $0^{\circ} \mathrm{C}$ and the lower boundary temperature as $647^{\circ} \mathrm{C}$ which is the contact temperature obtained from FTDM based on similarity solution (Carslaw and Jaeger 1959; Turcotte and Schubert 1982). The moving boundary positions are shown in figure 2(a) by dashed lines for the case CHSM, solid lines for the case FTDM, and symbols for the present case (FSM). In the case of CHSM with zero surface temperature, solidification front always moves downward. For FTDM, the dyke becomes completely solidified at time $t=0.0156$ m.y. For FSM, the non-uniform cooling causes the body to solidify
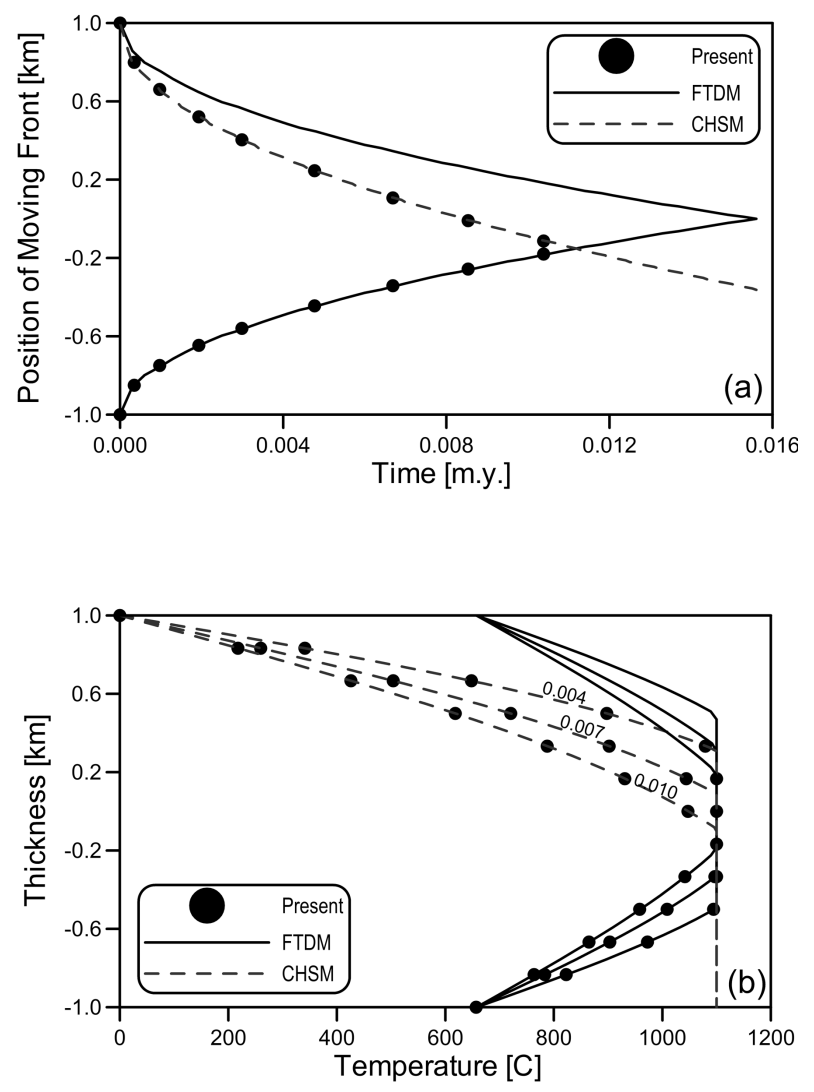

Figure 2. (a) Positions of moving boundaries (solid circles) and (b) corresponding thermal structure within the lava body at $0.004,0.007$, and $0.01 \mathrm{~m} . \mathrm{y}$. time during the nonuniform cooling of a $2 \mathrm{~km}$ thick lava body with the upper and lower contact temperatures at $0^{\circ} \mathrm{C}$ and $647^{\circ} \mathrm{C}$, respectively. These are compared with error function solutions for CHSM (dashed lines) and FTDM (solid lines). at $t=0.0111 \mathrm{~m} . \mathrm{y}$. The upper moving front in this case follows CHSM moving front and the lower moving front follows the lower segment of FTDM. Solidification of the body completes when these two fronts merge together, indicating that the two solutions CHSM and FTDM can together simulate the non-uniform cooling case provided the contact temperatures at the top surface and at the floor of the lava lake remain constant during the time of solidification. Corresponding temperature distributions within the body at time 0.004, 0.007, and 0.01 m.y. time also match well (figure $2(\mathrm{~b})$ ).

\subsection{Solidification of magma intrusion}

Solution for FTDM is based on the assumption that the country rock is initially at a constant temperature. However, in actual geological scenario any intrusive is subjected to a geothermal gradient due to the flow of heat from the interior of the earth. Therefore, contact temperature can be different (and possibly a function of time) from that obtained from similarity solution. In the second example, we consider such a scenario involving solidification of underplated magma. Manglik and Singh (1995) studied the problem of solidification of magma underplated at the base of the continental crust and showed that the contact temperature varies with time during the solidification due to the presence of geothermal gradient in the country rock. We obtained contact temperatures for one such case using their algorithm (referred subsequently as MS95 algorithm) and used these as boundary conditions in the second example to validate the present solution. In MS95 algorithm physical properties of magma intrusion and country rocks were assumed to be the same. In the present algorithm it is possible to include the effect of variation in physical properties of country rocks through variation in the values of contact temperatures.

Contact temperatures were calculated for the case of an underplating of $5 \mathrm{~km}$ thick melt at the base of $35 \mathrm{~km}$ thick continental crust. An exponential model of radiogenic heat sources distribution with surface heat generation $A_{0}=3.0 \mu \mathrm{W} / \mathrm{m}^{3}$ and characteristic depth $d=10 \mathrm{~km}$ was used. The initial geotherm for these parameters gives the temperature values of $435^{\circ} \mathrm{C}$ and $487^{\circ} \mathrm{C}$ at 35 and $40 \mathrm{~km}$ depths, respectively. Contact temperatures corresponding to these temperatures are $862^{\circ} \mathrm{C}$ and $885^{\circ} \mathrm{C}$, respectively, if similarity solution is used. Contact temperatures at the upper and lower boundary of the intrusive, obtained by MS95 algorithm, are shown in figure 3 by solid and dashed curves, respectively. Variation in contact temperatures at very short time is shown in the inset of 


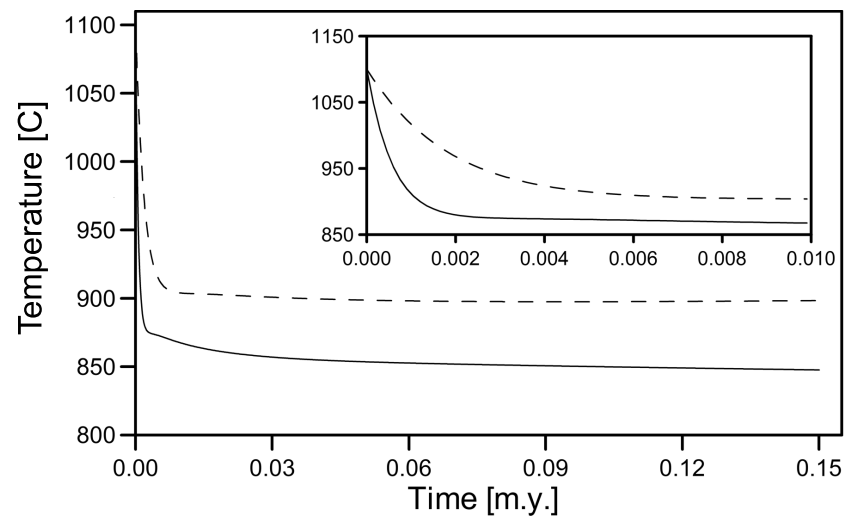

Figure 3. Variation in contact temperatures with time for the cooling of an underplated layer of $5 \mathrm{~km}$ thickness emplaced at the base of the continental crust of $35 \mathrm{~km}$ thickness. This is obtained by using MS95 algorithm. Dashed and solid curves represent the lower and the upper boundary contact temperatures, respectively.
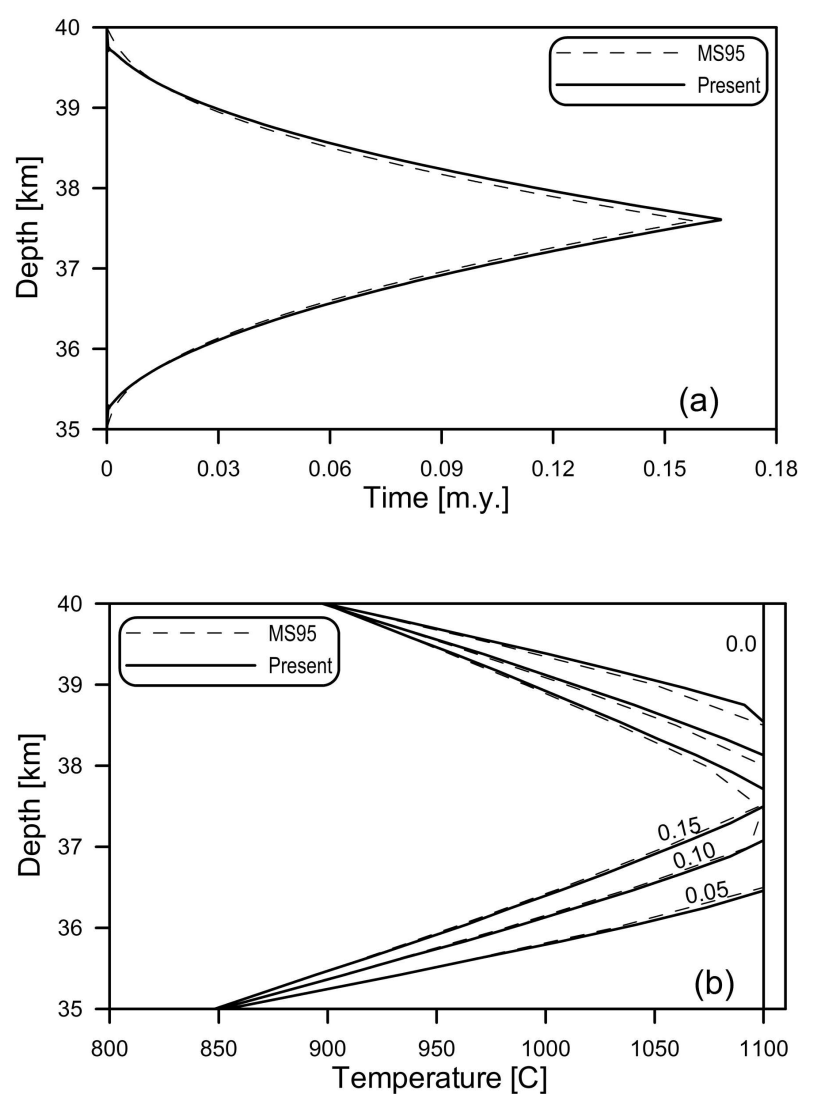

Figure 4. (a) Positions of solidification fronts and (b) corresponding thermal structure within the underplated layer at $0.0,0.05,0.1$, and $0.15 \mathrm{~m} . \mathrm{y}$. time for the time-varying contact temperatures shown in figure 3 . Solid curves are the results obtained by the present algorithm and the dashed curves are the results from MS95 algorithm.

the figure. At large times, contact temperatures are $848^{\circ} \mathrm{C}$ and $898^{\circ} \mathrm{C}$, respectively for the upper and the lower contacts. These values are slightly different from those obtained by similarity solution (FTDM). This time-temperature variation is used in the present algorithm to obtain the solidification time and thermal evolution within the intrusive. Moving boundary positions for this case are shown in figure 4(a) by solid lines. Dashed lines represent the positions from MS95 algorithm. These results are in good agreement. The results of thermal structure at $0.05,0.1$, and 0.15 m.y. are shown in figure 4(b). These results indicate that the present solution can be applied to calculate the thermal evolution within lava lakes and magma intrusions for a variety of contact temperature variations.

\section{Conclusions}

Lava lakes solidify by liberating heat to the surroundings at a non-uniform rate; the upper surface experiences fast convective removal of heat to the air/water whereas the lower surface releases heat to the basement by conductive mode of heat transfer. The rate of heat loss is also time-dependent. This is a Stefan problem with two moving solidification fronts separating the central melt zone from the solidified domains. The available analytical solution based on similarity variable method for cooling of a dyke gives symmetrical cooling with respect to the centre of the dyke and does not incorporate the effect of thermal gradient in the country rock. The present semi-analytical solution incorporates non-uniform cooling of lava lakes and magma intrusions. This can be used to analyse the effect of time-varying contact temperatures on the movement of solidification fronts and thermal evolution within the lava lake/magma intrusion. In this solution we have externally provided a variation of contact temperatures with time and obtained thermal evolution within the lava lake and magma body. This time variation of contact temperatures can as well be obtained by coupling the boundaries of the magma body with the surrounding country rocks through continuity of temperature and heat flux conditions. The present solution, however, incorporates only the conduction mode of heat transfer. In the case of thick lava lakes the thermal boundary layer formed at the top surface due to cooling can become unstable, leading to convective mode of heat transfer.

\section{Acknowledgements}

I am thankful to two anonymous reviewers for their encouraging reviews. Permission from the Director, NGRI, to publish this work is gratefully acknowledged. 


\section{References}

Carslaw H S and Jaeger J C 1959 Conduction of Heat in Solids. 2nd Edn (New York: Oxford Univ. Press) p. 510.

Cox K G 1993 Continental magmatic underplating; Phil. Trans. R. Soc. Lond. A342 155-166.

Delany P T 1987 Heat transfer during emplacement and cooling of mafic dykes; In: Mafic Dyke Swarms (eds) H C Halls and W F Fahrig Geol. Assoc. Can. Spec. Pap. $3431-36$.

Delany P T 1988 Fortran 77 programs for conductive cooling of dykes with temperature dependent thermal properties and heat of crystallization; Comput. Geosci. 14 181-212.

Gettings M E 1988 Variation of depth to the brittle-ductile transition due to cooling of a midcrustal intrusion; Geophys. Res. Lett. 153 213-126.

Gliko A O and Rovensky O N 1985 A numerical study of the process of lithospheric thinning under conditions of large heat flow; Izv. Akad. Nauk. SSSR Ser. Fiz. 21 416-419.

Griffiths R W and Fink J H 1992 Solidification and morphology of submarine lavas: A dependence on extrusion rate; J. Geophys. Res. 97 19729-19737.

Huppert H E 1990 The fluid mechanics of solidification; J. Fluid Mech. 212 209-240.

Huppert H E and Worster M G 1992 Vigorous motions in magma chambers and lava lakes; In: Chaotic Processes in the Geological Sciences (ed.) D A Yuen (New York: Springer-Verlag) Pp. 141-185.

Jaeger J C 1968 Cooling and solidification of igneous rocks; In: Basalts: The Poldervaart Treatise on Rocks of Basaltic Composition (eds) H H Hess and A Poldervaart (New York: Wiley-Interscience) Pp. 503-536.

Jaupart C and Tait S 1995 Dynamics of differentiation in magma reservoirs; J. Geophys. Res. 100 17615-17636.

Karner G D, Egan S S and Weissel J K 1992 Modeling the tectonic development of the Tucano and
Sergipe-Alagoas rift basin, Brazil; Tectonophys. 215 133-160.

Lightfoot N M H 1929 The solidification of molten steel; Proc. London Math. Soc. 31 97-116.

Lister J R 1995 Fluid-mechanical models of the interaction between solidification and flow in dykes; In: Physics and chemistry of dykes (eds) Baer and Heimann, Balkema and Rotterdam, Pp. 115-124.

Lister J R and Kerr R C 1991 Fluid-mechanical models of crack propagation and their application to magma transport in dykes; J. Geophys. Res. 96 10049-10077.

Manglik A and Singh R N 1995 Postintrusive thermal evolution of continental crust: A moving boundary approach; J. Geophys. Res. 100 18031-18043.

Manglik A, Ramana D V, Gliko A O and Singh R N 1992 Application of the Fourier method to the numerical solution of moving boundary problem in heat conduction; Proc. Ind. Acad. Sci. (EPS) 101 77-88.

Malamed V G 1958 Reducing Stefan's problem to a system of ordinary differential equation; Izv. Akad. Nauk. SSSR Ser. Geofis. 7 843-869.

Turcotte D L and Schubert G 1982 Geodynamics: Applications of Continuum Physics to Geological Problems (New York: John Wiley) p. 450.

White R S 1992 Magmatism during and after continental break-up; In: Magmatism and the Causes of Continental Break-up (eds) B C Storey, T Alabaster and R J Pankhurst Geol. Soc. Spec. Publ. 68 1-16.

Watson S and McKenzie D 1991 Melt generation by plumes: A study of Hawaiian volcanism; J. Petrol. 32 501-537.

White R S 1993 Melt production rates in mantle plumes; Phil. Trans. R. Soc. Lond. A342 137-153.

White R S and McKenzie D 1989 Magmatism at rift-zones: The generation of volcanic continental margins and flood basalts; J. Geophys. Res. 94 7685-7729.

Worster M G, Huppert H E and Sparks R S J 1993 The crystallization of lava lakes; J. Geophys. Res. 98 15891-15901. 